\title{
On the dynamics of positive solutions for the difference equation in a new population model
}

\author{
Wenjie Wang ${ }^{a, *}$, Hui Feng ${ }^{b}$ \\ ${ }^{a}$ Department of Mathematics, Lanzhou Jiaotong University, Lanzhou Gansu 730070, China. \\ ${ }^{b}$ Department of Mathematics, Northwest Normal University, Lanzhou Gansu 730070, China.
}

Communicated by B. G. Sidharth

\begin{abstract}
In this paper we study the boundedness and the asymptotic behavior of positive solutions for the difference equation

$$
x_{n+1}=a+b x_{n} \mathrm{e}^{-x_{n-1}},
$$

where $a, b$ are positive constants, and the initial values $x_{-1}, x_{0}$ are nonnegative numbers. (c)2016 All rights reserved.
\end{abstract}

Keywords: Difference equations, boundedness, asymptotic behavior.

2010 MSC: 34D05, 39A11.

\section{Introduction}

In recent years, the behavior of positive solutions for difference equation of exponential form has attracted great attention of many authors. In [3] in particular, Metwally studied the boundedness, the asymptotic behavior, the periodic character and the stability of solutions of the difference equation

$$
x_{n+1}=\alpha+\beta x_{n-1} \mathrm{e}^{-x_{n}},
$$

where $\alpha, \beta$ are positive constants and the initial values $x_{-1}, x_{0}$ are positive numbers. Later in [4], Fotiades studied the existence, uniqueness and attractivity of prime period two solution of this equation. For similar research on difference equation, we refer the reader to $[1,2,5,6,7,8$, and the references therein.

\footnotetext{
${ }^{*}$ Corresponding author

Email addresses: wangwenjie@mail.lzjtu.cn (Wenjie Wang), fh_9237@163.com (Hui Feng)
} 
Motivated by the above research, we will change the above equation to a naturally new form as

$$
x_{n+1}=a+b x_{n} \mathrm{e}^{-x_{n-1}} .
$$

In this paper, we will investigate the boundedness nature, the persistence and the asymptotic behavior of the positive solutions of (1.1), where the parameters $a, b$ are positive numbers and the initial value $x_{-1}, x_{0}$ are arbitrary nonnegative numbers.

Equation (1.1) could be also viewed as a model in mathematical biology, in which case we consider $a$ as the immigration rate and $b$ as the population growth rate of one species $x_{n}$. Observe that it is very crucial that every solution of (1.1) should be bounded since the population of species $x_{n}$ can not grow infinitely due to the limited resources. In addition, the equilibrium point of 1.1 is considered to be the natural ideal population.

\section{Boundedness, persistence and asymptotic behavior of positive solutions of (1.1)}

Firstly, we establish the existence and uniqueness of equilibrium of (1.1).

Proposition 2.1. Equation (1.1) has a unique positive equilibrium $\bar{x}$.

Proof. Observe that the equilibrium points of (1.1) are the solutions of the equation

$$
\bar{x}=a+b \bar{x} \mathrm{e}^{-\bar{x}}
$$

Set

$$
g(x)=a+b x \mathrm{e}^{-x}-x .
$$

Then

$$
g(0)=a, \lim _{x \rightarrow \infty} g(x)=-\infty
$$

and

$$
g^{\prime}(x)=b \mathrm{e}^{-x}(1-x)-1
$$

It suffices to show that

$$
g^{\prime}(x)<0
$$

Now

$$
g^{\prime}(\bar{x})=b \mathrm{e}^{-\bar{x}}(1-\bar{x})-1
$$

and $\bar{x}>a$, so

$$
g^{\prime}(\bar{x})<0 .
$$

As $g^{\prime}$ is continuous, there exists an $\varepsilon$ such that for $x \in(\bar{x}-\varepsilon, \bar{x}+\varepsilon)$

$$
g^{\prime}(x)<0 .
$$

Therefore from $(2.2), g$ is decreasing in the interval $(\bar{x}-\varepsilon, \bar{x}+\varepsilon)$. Suppose that $g$ has roots greater than the root $\bar{x}$. Let $x_{1}$ be the smallest root of $g$ such that $x_{1}>\bar{x}$. Similar to the argument above, we can show that there exists an $\varepsilon_{1}$ such that $g$ is decreasing in the interval $\left(x_{1}-\varepsilon_{1}, x_{1}+\varepsilon_{1}\right)$. Since $g(\bar{x}+\varepsilon)<0, g\left(x_{1}-\varepsilon_{1}\right)>0$ and $g$ is continuous, we see that $g$ must have a root in the interval $\left(\bar{x}+\varepsilon, x_{1}-\varepsilon_{1}\right)$. This is clearly a contradiction since $x_{1}$ is the smallest root of $g$ such that $x_{1}>\bar{x}$. Similarly we can prove that $g$ has no solutions in $(a, \bar{x})$. Therefore equation $g(x)=0$ must have a unique solution in $(a,+\infty)$. So (1.1) has exactly one solution $\bar{x}$, and furthermore $\bar{x}>a$.

The following proposition gives a sufficient condition for every positive solution of (1.1) to be bounded. 
Proposition 2.2. Every positive solution of (1.1) is bounded if

$$
b<\mathrm{e}^{a} .
$$

Proof. Let $\left\{x_{n}\right\}_{n=-1}^{\infty}$ be an arbitrary solution of (1.1). Observe that for all $n \geq 2$,

$$
x_{n+1}=a+b x_{n} \mathrm{e}^{-x_{n-1}} \leq a+b x_{n} \mathrm{e}^{-a} .
$$

We will now consider the non-homogeneous difference equations

$$
y_{n+1}=a+b y_{n} \mathrm{e}^{-a}, n=2,3, \cdots .
$$

Therefore, from (2.5), an arbitrary solution $\left\{y_{n}\right\}_{n=-1}^{\infty}$ of 2.5 is given by

$$
y_{n}=r\left(b \mathrm{e}^{-a}\right)^{n}+\frac{a}{1-b \mathrm{e}^{-a}},
$$

where $r$ depends on the initial values $y_{-1}$. Thus we see that relations $(2.3)$ and $(2.6)$ imply that $\left\{y_{n}\right\}$ is bounded sequences. Now we will consider the solution $y_{n}$ of $(2.5)$ such that

$$
y_{2}=x_{2} .
$$

Thus from (2.4), (2.5) and (2.7) we get

$$
x_{n+1}-y_{n+1} \leq b\left(x_{n}-y_{n}\right) \mathrm{e}^{-a},
$$

and by induction, we have

$$
x_{n} \leq y_{n} \text { for all } n \geq 1 .
$$

Therefore it follows that $\left\{x_{n}\right\}$ is a bounded sequence. Hence the proof of the proposition is complete.

In the next proposition we will study the existence of invariant intervals of (1.1).

Proposition 2.3. Consider (1.1) where relation (2.3) hold. Then the following statements are true:

(i) The set

$$
\left[a, \frac{a}{1-b \mathrm{e}^{-a}}\right]
$$

is an invariant set for (1.1).

(ii) Let $\varepsilon$ be an arbitrary positive number and $x_{n}$ be an arbitrary solution of (1.1). We then consider the set

$$
I=\left[a, \frac{a+\varepsilon}{1-b \mathrm{e}^{-a}}\right]
$$

Then there exists an $n_{0}$ such that for all $n \geq n_{0}$

$$
x_{n} \in I \text {. }
$$

Proof. (i) Let $x_{n}$ be a solution of (1.1) with initial values $x_{-1}, x_{0}$ such that

$$
x_{-1}, x_{0} \in\left[a, \frac{a}{1-b \mathrm{e}^{-a}}\right] .
$$

Then from (1.1) and 2.10 we get

$$
a \leq x_{1}=a+b x_{0} \mathrm{e}^{-x_{-1}} \leq a+b \frac{a}{1-b \mathrm{e}^{-a}} \mathrm{e}^{-a}=\frac{a}{1-b \mathrm{e}^{-a}} .
$$


Then it follows by induction that

$$
a \leq x_{n} \leq \frac{a}{1-b \mathrm{e}^{-a}}, n=1,2, \cdots .
$$

This completes the proof of (i).

(ii) Let $x_{n}$ be an arbitrary solution of (1.1). Therefore, from Proposition 2.2 we assume

$$
0<l=\liminf _{n \rightarrow \infty} x_{n}, L=\limsup _{n \rightarrow \infty} x_{n}<\infty .
$$

It follows from (1.1) and 2.11) that

$$
L \leq a+b L \mathrm{e}^{-l}, l \geq a+b l \mathrm{e}^{-L},
$$

which imply that

$$
a \leq L \leq \frac{a}{1-b \mathrm{e}^{-a}} .
$$

Thus from (1.1), we see that there exists an $n_{0}$ such that 2.9 holds true. This completes the proof of (ii).

Before stating our main result, we next give the following lemma which is a minor modification of Theorem 1.11 in [5].

Lemma 2.4. Consider the difference equation

$$
y_{n+1}=f\left(y_{n}, y_{n-1}\right), n=0,1, \cdots .
$$

Let $f:[a, b] \times[a, b] \longrightarrow[a, b]$ be a continuous function and $a, b$ be positive numbers. Suppose $f$ satisfies the following properties:

a.) $f(u, v)$ is nondecreasing in $u$ and nonincreasing in $v$;

b.) If $(m, M) \in[a, b] \times[a, b]$ is a solution of the system

$$
m=f(m, M) \text { and } M=f(M, m),
$$

then $m=M$.

Then 2.12 has a unique positive equilibrium $\bar{y}$ and every positive solution of 2.12 converges to $\bar{y}$.

Proof. Set

$$
m_{0}=a \text { and } M_{0}=b
$$

and for $i=1,2, \cdots$, set

$$
m_{i}=f\left(m_{i-1}, M_{i-1}\right), \quad M_{i}=f\left(M_{i-1}, m_{i-1}\right) .
$$

Now observe that

$$
m_{0} \leq m_{1} \leq \cdots \leq m_{i} \leq \cdots \leq \cdots \leq M_{i} \leq \cdots \leq M_{1} \leq M_{0}
$$

and

$$
m_{i} \leq y_{n} \leq M_{i} \text { for } n \geq 2 i-1 \text {. }
$$

Set

$$
m=\lim _{n \rightarrow \infty} m_{i}, \quad M=\lim _{n \rightarrow \infty} M_{i} .
$$

Clearly,

$$
m \leq \liminf _{n \rightarrow \infty} y_{i} \leq \limsup _{n \rightarrow \infty} y_{i} \leq M
$$

and by the continuity of $f$,

$$
m=f(m, M), \quad M=f(M, m),
$$

and thus $m=M=\bar{y}$. The proof is complete. 
In the next proposition we will study the asymptotic behavior of positive solutions of (1.1).

Proposition 2.5. Consider (1.1) where the initial values $x_{-1}, x_{0}$ are positive constants and $a, b$ are nonnegative constants satisfying

$$
b<\mathrm{e}^{a} \frac{-a+\sqrt{a^{2}+4}}{2} .
$$

Then (1.1) has a unique positive equilibrium $\bar{x}$ such that

$$
\bar{x} \in\left[a, \frac{a}{1-b \mathrm{e}^{-a}}\right] .
$$

Moreover every positive solution of (1.1) tends to the unique positive equilibrium $\bar{x}$ as $n \longrightarrow \infty$.

Proof. Equation (1.1) has a unique positive equilibrium such that relation (2.14) holds follows by Proposition 2.1 and Proposition 2.2

It suffices to show that any positive solution $\left\{x_{n}\right\}$ converges to the unique positive equilibrium $\bar{x}$ of (1.1). We consider the function

$$
f(x, y)=a+b x \mathrm{e}^{-y}, \quad x, y \in I,
$$

where $I$ is defined in 2.8 . Then from $(2.3)$ and (2.15), we see that for $x, y \in I$

$$
a \leq f(x, y) \leq a+b \frac{a+\varepsilon}{1-b \mathrm{e}^{-a}} \mathrm{e}^{-a}=\frac{a+b \varepsilon \mathrm{e}^{-a}}{1-b \mathrm{e}^{-a}}<\frac{a+\varepsilon}{1-b \mathrm{e}^{-a}}
$$

and so $f: I \times I \longrightarrow I$. Let $x_{n}$ be an arbitrary solution of (1.1). Therefore, as 2.13) implies (2.3), from Proposition 2.3, there exists an $n_{0}$ such that 2.9 holds true. Let $m, M$ be positive real numbers such that

$$
M=a+b M \mathrm{e}^{-m}, m=a+b m \mathrm{e}^{-M} .
$$

From (2.16), it follows that

$$
M=\ln \frac{b m}{m-a}, m=\ln \frac{b M}{M-a} .
$$

Thus we see that 2.16) and (2.17) imply

$$
\ln \left(\frac{b m}{m-a}\right)\left(1-b \mathrm{e}^{-m}\right)=a, \ln \left(\frac{b M}{M-a}\right)\left(1-b \mathrm{e}^{-M}\right)=a .
$$

We then consider the function

$$
F(x)=\ln \left(\frac{b x}{x-a}\right)\left(1-b \mathrm{e}^{-x}\right)-a .
$$

Let $z$ be a solution of $F(x)=0$. We claim that

$$
F^{\prime}(z)<0
$$

From (2.18) we see that

$$
F^{\prime}(z)=\left(1-b \mathrm{e}^{-z}\right) \frac{-a}{z(z-a)}+\ln \left(\frac{b z}{z-a}\right) b \mathrm{e}^{-z} .
$$

Since $z$ satisfies equation $F(x)=0$, then it follows that

$$
\ln \left(\frac{b z}{z-a}\right)=\frac{a}{1-b \mathrm{e}^{-z}} .
$$

Therefore, relations (2.20) and (2.21) imply that

$$
F^{\prime}(z)=\left(1-b \mathrm{e}^{-z}\right) \frac{-a}{z(z-a)}+\frac{a b \mathrm{e}^{-z}}{1-b \mathrm{e}^{-z}} .
$$


Using (2.22), to prove our claim 2.19), it suffices to prove that

$$
H(z)-G(z)<0
$$

where

$$
H(z)=b z(z-a), G(z)=\mathrm{e}^{z}\left(1-b \mathrm{e}^{-z}\right)^{2} .
$$

From 2.24) we get

$$
\begin{aligned}
& H^{\prime}(z)=b(2 z-a), G^{\prime}(z)=-b^{2} \mathrm{e}^{-z}+\mathrm{e}^{z}, \\
& H^{\prime \prime}(z)=2 b, G^{\prime \prime}(z)=b^{2} \mathrm{e}^{-z}+\mathrm{e}^{z} \\
& H^{\prime \prime \prime}(z)=0, G^{\prime \prime \prime}(z)=-b^{2} \mathrm{e}^{-z}+\mathrm{e}^{z} .
\end{aligned}
$$

Now from (2.13) and (2.25), we see that as $z>a$ we have

$$
H^{\prime \prime \prime}(z)-G^{\prime \prime \prime}(z)<0
$$

Since $z>a$, we have

$$
H^{\prime \prime}(z)-G^{\prime \prime}(z)<H^{\prime \prime}(a)-G^{\prime \prime}(a)=2 b-b^{2} \mathrm{e}^{-a}-\mathrm{e}^{a}=-\mathrm{e}^{-a}\left(b-\mathrm{e}^{a}\right)^{2}<0 .
$$

Therefore from $(2.26)$ and since $z>a$, it follows

$$
H^{\prime}(z)-G^{\prime}(z)<H^{\prime}(a)-G^{\prime}(a)=a b+b^{2} \mathrm{e}^{-a}-\mathrm{e}^{a}=\mathrm{e}^{-a}\left(b^{2}+a b \mathrm{e}^{a}-\mathrm{e}^{2 a}\right) .
$$

Now observe that from $(2.13)$ we have

$$
b^{2}+a b \mathrm{e}^{a}-\mathrm{e}^{2 a}<0 .
$$

Therefore relations 2.27 and $(2.28)$ imply that

$$
H^{\prime}(z)-G^{\prime}(z)<0 .
$$

Hence from 2.29 and as $z>a$, we get

$$
H(z)-G(z)<H(a)-G(a)<0,
$$

which implies that (2.19) is true. By continuity of $F^{\prime}$, it is known that there exists an $\varepsilon$ such that for $x \in(z-\varepsilon, z+\varepsilon)$

$$
F^{\prime}(x)<0 .
$$

Therefore from (2.31), the function $F$ is decreasing in the interval $(z-\varepsilon, z+\varepsilon)$. Suppose that $F$ has roots greater than the root $z$. Let $z_{1}$ be the smallest root of $F$ such that $z_{1}>z$. Similar to the argument above, we can show that there exists an $\varepsilon_{1}$ such that $F$ is decreasing in the interval $\left(z_{1}-\varepsilon_{1}, z_{1}+\varepsilon_{1}\right)$. Since $F(z+\varepsilon)<0, F\left(z_{1}-\varepsilon_{1}\right)>0$ and $F$ is continuous, we see that $F$ must have a root in the interval $\left(z+\varepsilon, z_{1}-\varepsilon_{1}\right)$. This is clearly a contradiction since $z_{1}$ is the smallest root of $F$ such that $z_{1}>z$. Similarly we can prove that $F$ has no solutions in $(a, z)$. Therefore equation $F(x)=0$ must have a unique solution. Hence from (2.17) and 2.18), we obtain $m=M$. By Lemma 2.4, the proof of the proposition is complete.

Example 2.6. See figure (a), shows the stability of equilibrium of 1.1 and 1(b) shows the unstable case whenever 2.13$)$ is not satisfied. 


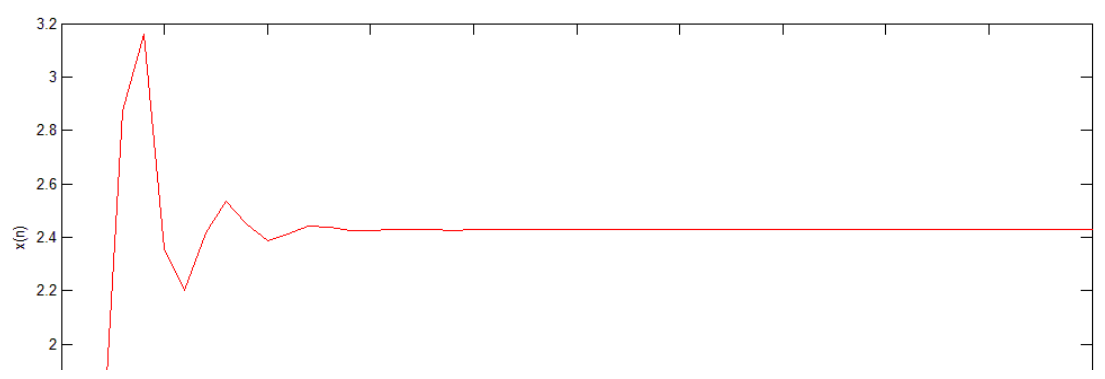

(a) $a=2, b=2$.

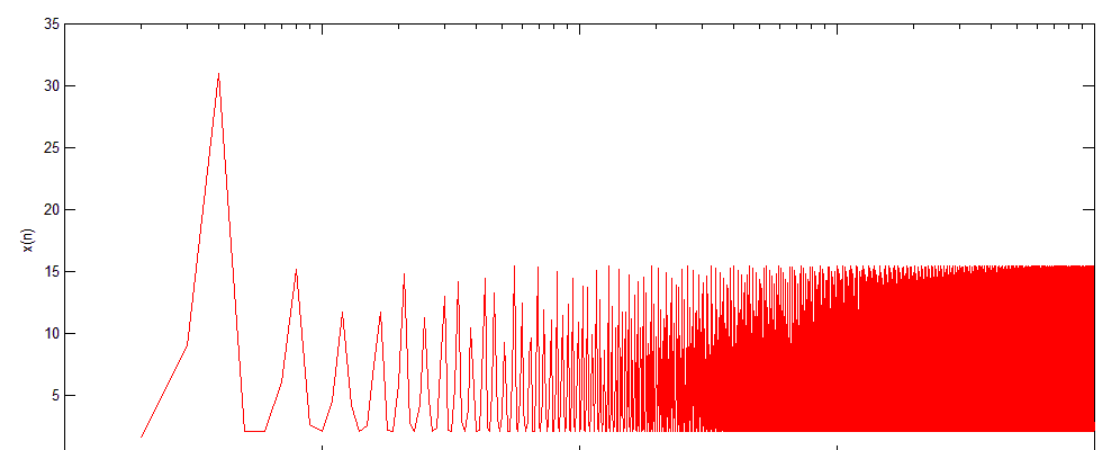

(b) $a=4, b=16$.

Figure 1

\section{Acknowledgements}

This paper was funded by the National Natural Science Foundation of China(61363058), Natural Science Foundation of Gansu Province(145RJZA232,145RJYA259) and Promotion Funds for Young Teachers in Northwest Normal University (NWNU-LKQN-12-14).

\section{References}

[1] J. R. Beddington, C. A. Free, J. H. Lawton, Dynamic complexity in predator prey models framed in difference equations, Nature, 255 (1975) 58-60.1

[2] R. Devault, W. Kosmala, G. Ladas, S. W. Schultz, Global Behavior of $y_{n+1}=\frac{p+y_{n-k}}{q y_{n}+y_{n-k}}$, Nonlinear Anal., 47 (2001), 4743-4751.1.

[3] E. El-Metwally, E. A. Grove, G. Ladas, R. Levins, M. Radin, On the difference equation $x_{n+1}=\alpha+\beta x_{n-1} e^{-x_{n}}$, Nonlinear Anal., 47 (2001), 4623-4634.1

[4] N. Fotiades, G. Papaschinopoulos, Existence, uniqueness and attractivity of prime period two solution for a difference equation of exponential form, Appl. Math. Comput. Model., 218 (2012), 11648-11653.1.

[5] V. L. Kocic, G. Ladas, Global Behavior of Nonlinear Difference Equations of Higher Order with Applications, Kluwer Academic Publisher, Dordrecht, (1993).1.2

[6] G. Papaschinopoulos, M. Radin, C. J. Schinas, On the system of two difference equations of exponential form: $x_{n+1}=a+b x_{n-1} e^{-y_{n}} ; y_{n+1}=c+d y_{n-1} e^{-x_{n}}$, Math. Comput. Model., 54 (2011), 2969-2977.1

[7] G. Stefanidou, G. Papaschinopoulos, C. J. Schinas, On a system of two exponential type difference equations, Comm. Appl. Nonlinear Anal., 17 (2010), 1-13.1

[8] I. Yalcinkaya, On the global asymptotic behavior of a system of two nonlinear difference equations, Ars Combin., 95 (2010), 151-159.1 